\title{
Direct Method of Calculus of Variations in Elasticity
}

\author{
Alessandro Della Corte ${ }^{1,2}$ and Francesco \\ dell'Isola ${ }^{3}$ \\ ${ }^{1}$ DIMA, University of Rome La Sapienza, \\ Rome, Italy \\ ${ }^{2}$ International Research Center M\&MoCS, \\ University of L'Aquila, L'Aquila, Italy \\ ${ }^{3}$ MEMOCS Centre Università dell'Aquila, DISG \\ Università di Roma La Sapienza, Rome, Italy
}

\section{Definition}

Theorems on the existence of minimizers for functionals defined on Banach spaces, and related approximation methods, applied to minimization problems arising in elasticity theory.

\section{Introduction}

Variational methods are a powerful tool in elasticity and in fact the only known approach able to guarantee sufficient generality in the treatment of problems arising in hyperelasticity (see the corresponding entry), in the asymptotic derivation of two-dimensional elastic models (Ciarlet $1988,1997)$, and in many other cases in continuum mechanics (Pedregal 2000; Fonseca 1987; dell'Isola and Placidi 2011). Variational problems take usually the form of a minimization problem that can be described as follows: we search the minimum of a functional $F(u)$ defined on a subset $S$ of a Banach space $B$ (i.e., a normed, complete vector space) and taking values in $[-\infty,+\infty]$. Direct method provides sufficient conditions on $S, B$, and $F$ for the existence of a minimizer $\tilde{u} \in S$. The main ideas of the method were developed by Leonida Tonelli at the beginning of twentieth century (Tonelli 1911, 1920). 
The problem described above presents clear similarities with the one concerning the existence of extremal points for real functions of $n$ variables. The simplest result in this direction is represented by the theorem stating that a continuous function defined on a closed, bounded domain of $\mathbb{R}^{n}$ attains its minimum and maximum. At a slightly higher level of abstractness, the natural generalization of closed, bounded sets in $\mathbb{R}^{n}$ is compact sets (in fact Heine-Borel theorem guarantees that the two concepts are equivalent in $\mathbb{R}^{n}$ if "bounded" is intended with respect to the Euclidean distance). The well-known Weierstrass extreme value theorem ensures, indeed, that continuous real functions defined on compact sets have a minimum (and a maximum). Unfortunately, in infinite-dimensional Banach spaces (which are naturally involved in the mathematical formulation of elasticity), closedness and boundedness are far from sufficient conditions for compactness with respect to the topology induced by the norm, and in fact it is well known that the unit ball is not totally bounded in Banach spaces (i.e., it cannot be covered by a finite number of open balls of any given radius). This implies that a model-case variational problem formulated in integral terms:

$$
\min _{u \in S} \int_{C} f(\boldsymbol{x}, u, \nabla u)
$$

cannot benefit from a direct application of the extreme value theorem above cited in the case in which $S$ is the unit ball in $B$ or any homeomorphic image of it. (We recall that compactness is a topological invariant.)

The basic idea of direct method is the following: we can introduce a new topology in $B$ in which the domain of the functional is compact or at least enjoys some key properties of compact sets. Recalling the definition of compactness, (A set is said to be compact if each of its open covers admits a finite subcover.) it is clear that, to this aim, we have to "reduce" the open sets, i.e., the open sets in the new topology should be a subset of the open sets of the topology induced by the norm. On the other hand, we must still have "enough" open sets to make the functional $F$ continuous, or at least to preserve the characteristics of continuity that play a role in the proof of the existence of a minimum. The problem is then to balance between these two opposite requirements, and it is solved by introducing the so-called weak and weak* topology in $B$, as we will briefly describe in the following section. The topology induced by the norm in $B$ is called instead the strong topology.

Before describing the method in some of its technical aspects, we note that problems such as (1) appear in classical Cauchy elasticity, $C$ being obviously the reference configuration of an elastic body, $x$ a point in the Euclidean threedimensional affine space, and $u(x)$ the placement (or displacement) function. The mathematical treatment of these problems has been one of the main stimuli for the birth of the direct method of calculus of variations.

\section{The Method and Its Applications to Elasticity}

\section{Outline of the Method}

The technical treatment of the direct method applied to elasticity problems, especially in 3D, requires concepts from the general theory of Sobolev spaces (Sobolev 1950), as well as some quite refined notions of convex and quasiconvex analysis. Here we just want to summarize the relevant technical features of the method without trying to provide rigorous justifications of all the statements. For a rigorous and comprehensive treatment, the reader is referred to the excellent treatises Dacorogna (2007) and notably for the general theory in $L^{p}$ spaces Fonseca and Leoni (2007).

To implement the ideas introduced in the previous section, it is convenient to reformulate the compactness properties in terms of sequences. It is well known that a compact set in $\mathbb{R}^{n}$ has the property that every infinite sequence contained in 
it has a converging subsequence (this property is called sequential compactness). In metric spaces, the concepts of compactness and sequential compactness are equivalent, and therefore one can address compactness considering the behavior of sequences rather than the question of the existence of finite open subcovers (which is in general more difficult to check). More specifically, one can consider minimizing sequences, (In French literature (e.g., in Ciarlet 1988), it is sometimes used the more logical name infimizing sequence.) i.e., sequences of functions $u_{n} \in S$ such that:

$$
\lim _{n \rightarrow \infty} F\left(u_{n}\right)=\inf _{u \in S} F(u)
$$

Such kind of sequences in general do not have any subsequence which is convergent, in the strong topology, to an element of $S$, i.e., there is in general no $u \in S$ such that $\left\|u_{n}-u\right\| \rightarrow 0$. However, one can consider the convergence in the following (weaker) sense: a sequence $u_{k}$ is said to converge to $u$ iff $\left\langle u_{k}, v *\right\rangle \rightarrow\left\langle u, v^{*}\right\rangle$ for every $v^{*} \in B^{*}$. (Here $B^{*}$ denotes the dual space of $B$, i.e., the vector space of continuous linear functionals on $B$, and $\left\langle a, b^{*}\right\rangle$ denotes the action of the continuous linear functional $b^{*} \in B^{*}$ on $a \in B$. Note that in a Hilbert space $H$ (e.g., the Sobolev spaces $\left.H^{k}:=W^{k, 2}\right),\left\langle a, b^{*}\right\rangle$ can be expressed by means of an inner product $\langle a, b\rangle$ with $b \in H$.) The convergence so introduced is called the weak convergence, and it can be proven (see, e.g., Rudin 1991) that this means convergence in the coarsest topology in $B$ such that all the elements of $B^{*}$ are still continuous. This topology is called the weak topology, and in the infinite-dimensional case, it has strictly fewer open sets than the strong topology; in particular, open balls in $B$ are not open sets in the weak topology. A further concept that is needed in the general case is that of weak* convergence, which can be defined as the pointwise convergence for linear functionals in $B^{*}$. It can be shown (see Rudin 1991) that, in $B^{*}$, weak convergence is implied by strong convergence (By this we mean the convergence induced by the usual functional norm $\left\|v^{*}\right\|_{B^{*}}:=\sup \left\langle u, v^{*}\right\rangle:\|u\|=1$.) and implies weak* convergence. The well-known Banach-Alaoglu theorem (in its sequential form) provides us the needed compactness property for bounded domains. Indeed, according to the theorem, any infinite sequence contained in the closed unit ball in $B^{*}$ (and therefore in every homeomorphic image of it) has a subsequence that is convergent in the weak* topology (Banach 1932; Alaoglu 1940). This is generally sufficient to allow the desired generalization of Weierstrass theorem. Notice indeed that in most applications to elasticity, the variational problems live naturally in Sobolev spaces of the type $W^{k, 2}$. Being Hilbert spaces, those spaces are always reflexive, i.e., they are canonically isomorphic to the dual space of their dual space, so that we can identify $B$ with $B^{* *}$.

Concerning the needed continuity assumptions, it has to be noted that in general in the weak and weak* topologies, one cannot rely on continuity for functionals having the form (1). (In fact, the same can be said for generic integral functionals.) A very simple (and effective) remark from Tonelli was that, in fact, there is no need of continuity for existence of minimizers in Weierstrass theorem. Indeed it is sufficient to establish the lower semicontinuity of a real function to ensure that it attains its minimum on a compact set. (A real function $f$ defined on a topological space $X$ is called lower semicontinuous if the set $\{x \in X: f(x)>\alpha\}$ is an open real set for every $\alpha \in \mathbb{R}$. A well-known example of a functional which is lower semicontinuous but not continuous is the arc-length functional, i.e., $L(f):=$ $\int_{a}^{b} \sqrt{1+\left(f^{\prime}(x)\right)^{2}} d x$. It is geometrically evident that, in the $C^{0}$ norm, $L$ is not continuous, as we can find functions arbitrarily close to a given rectifiable function whose graph has arbitrarily large length. On the other hand, it is easy to see that it is lower semicontinuous. This simple example shows how natural the concept of lower semicontinuity is. Clearly a similar result holds true for the existence of maxima of upper semicontinuous functions.) 
Having said this, the application of the direct method can be summarized as follows:

1. Prove that $F$ is bounded from below

2. Prove that a minimizing sequence $u_{n}$ has a subsequence $u_{n_{k}}$ which converges (in general in a weaker topology $T$ ) to an element $\tilde{u} \in S$

3. Prove that $F$ is lower semicontinuous with respect to the same topology $T$ used at step 2

We have then:

$$
\inf _{u \in S} F(u) \leq F(\tilde{u}) \leq \lim _{k \rightarrow \infty} F\left(u_{n_{k}}\right)=\inf _{u \in S} F(u)
$$

the last inequality holding because of the elementary properties of lower semicontinuity. Therefore, from (3), it is possible to conclude that $\tilde{u}$ is a minimizer for $F$ in $S \subseteq B$. It is important to understand that, in this procedure, the topology $T$ plays a mere "intermediate" role and in principle can be replaced by any other topology for which the needed continuity and compactness properties are satisfied at the same time.

Note that, in order to prove step 2, one needs to prove that the sequence $u_{n}$ is bounded (so as to be able to use the sequential Banach-Alaoglu theorem cited above to deduce the existence of $u_{n_{k}}$ ). To achieve this result, it is commonly asked some additional condition on $F$, the simplest case being the coercivity condition: $F(u) \rightarrow \infty$ as $\|u\| \rightarrow \infty$. In general, it is more difficult to prove the (sequential) weak lower semicontinuity of the functional. To this aim, a large chapter of functional analysis is devoted to the study of the continuity properties of integral functionals (a seminal work is Berkovitz 1974). The simplest result in this direction (which is a direct generalization of the famous Tonelli's theorem for $L^{p}$ spaces) is that, under suitable assumptions, the functional $F$ is lower semicontinuous if the integrand $f$ appearing in (1) is convex with respect to the variable $\nabla u$. In case of functions defined on a domain $\Omega \subset \mathbb{R}^{n}$ and taking values in $\mathbb{R}^{m+n}(n, m>1)$, one can weaken the convexity condition introducing the concepts of quasi- convexity and polyconvexity (works of historical significance are Morrey (1952) and Ball (1976), the latter focusing on problems in nonlinear elasticity; for a modern comprehensive treatment, we refer to Dacorogna (2007), Chapters 5-8). These kind of problems occur very often in nonlinear elasticity, the simplest example probably being that of inviscid compressible fluids where the energy density is a function of $\operatorname{det} \nabla u$.

\section{Role in Relaxation Theory}

Relaxation theory is a very important area in calculus of variations, and direct method constitutes a crucial element in it. The convexity conditions needed on the integrand of (1) (or also integral functionals with higher-order gradients) to ensure the weak lower semicontinuity of $F$ are not always satisfied when one introduces an energy model to describe a concrete elastic object. In some cases, non-convex (nonquasiconvex) integrals produce phenomena, such as arbitrarily oscillating solutions (the first examples were provided in Young 1937, 1938; McShane et al. 1940), which cannot be concretely realized by a physical system and, in any case, typically fail to satisfy wellposedness properties even in a very weak sense. To illustrate this, consider the elementary case of an integral functional $J(u)=\int_{C} f(u)$ when $f$ is a scalar function and there exist two points $u_{1}, u_{2}$ in $C$ such that $f\left(u_{1}\right)=f\left(u_{2}\right)<f(u) \quad \forall \quad u \in$ $\mathbb{R} \backslash\left\{u_{1}, u_{2}\right\}$. It is clear that partitioning the domain $C$ in any possible way in two disjoint measurable sets $C_{1}$ and $C_{2}$, every function $u$ taking the value $u_{i}$ on $C_{i}(i=1,2)$ will be a minimizer of $J$. In particular, if $C$ is a real interval, the function $u$ can oscillate arbitrarily often between the values $u_{i}$. On the other hand, it is also reasonable to think that, if the function $u$ describes some physical quantity susceptible of measurement, we will never observe an experimental datum with a non-countable number of oscillations (rather some average value, in a suitable sense, between $u_{1}$ and $u_{2}$ ).

The classical approach to deal with these problems is to introduce a relaxed energy density functional (a reference text on relaxation theory is Roubicek 1997), i.e., a functional $F^{\prime}$ such that: 
1. $F^{\prime}$ is sequentially weakly lower semicontinuous

2. $F^{\prime}(u) \leq F(u)$

3. $F^{\prime}$ is the largest functional verifying 1 . and 2 .

The conditions 2. and 3. guarantee that $\min F^{\prime}$ (if it exists) $=\inf F$, and condition 3 . also guarantees that $F^{\prime}$ has the desired growth condition to make sure that minimizing sequences are bounded. Therefore, direct method ensures that $F^{\prime}$ has a minimizer, and in many senses, the relaxation of the original problem from the functional $F$ to $F^{\prime}$ is the most sensible one.

In relaxation theory, a key role is played by the concepts of convex envelope and Fenchel conjugate (Dacorogna 2007), and the study of the integral representation of relaxed functionals is by now an important chapter in convex and quasiconvex analysis. In turn, relaxation theory has been able to cast light on some interesting phenomena occurring in calculus of variations that were poorly understood before (see, e.g., Belloni 1995 and, for results concerning problems in fracture mechanics, Bouchitté et al. 1995).

\section{Direct Method as an Approximation Method}

Direct method not only provides sufficient conditions for the existence of minimizers of variational problems, but may also be used to implement an approximation procedure to construct the solution of the problem (Gelfand and Fomin 1963). This procedure presents the advantage, when compared to the approximated solution of the corresponding Euler-Lagrange equations, to be able to approximate the solution even when it fails to verify the regularity conditions needed for the Euler-Lagrange equations. This is particularly relevant in elasticity theory, as it is well known that (especially in the nonautonomous case) often the solutions have weak regularity properties already in dimension one, and even when the integrand is seemingly simple, e.g., of polynomial type (see, for instance, Ball and Mizel 1987). The well-known approximation method for boundary value problems by Ritz (1908) and its generalization due to Galerkin (1915) base their convergence on the existence of converging minimizing subsequences. As a reference book on the related numerical method, the reader is referred to Axelsson and Barker (2001) (see also the entry Finite Element Method). Important results on the theoretical convergence of the methods by Ritz and Galerkin have been obtained by the soviet school (Kantorovich and Krylov 1962; Mikhlin 1957). An elementary application of the method, providing the explicit computation of the approximating sequence, can be found in Smirnov (2014), Vol. 4 Part 1 (see example 109).

\section{A Model-Case Application in 1D Nonlinear Elasticity}

As a model case of application of direct method, we can consider a 1D problem in nonlinear elasticity that leads to a nonautonomous variational problem. Let us consider an Euler inextensible Elastica of length $L$, clamped at one side and submitted to a uniformly distributed transverse load in large deformation regime. The corresponding variational problem can be rewritten in terms of the angular variable $\theta$ denoting the angle formed by the tangent to the Elastica and a reference axis (see Della Corte et al. 2017):

$$
\min _{\theta \in W^{1,2}: \theta(0)=0} E(\theta) \quad E(\theta):=\frac{1}{2} \int_{0}^{L}\left[\theta^{\prime}(s)^{2}-b(L-s) \sin \theta(s)\right] \mathrm{d} s
$$

where $b$ is the modulus of the distributed load. In a sense, this problem represents the simplest case in which nontrivial solutions can appear, as it is one-dimensional, it is nonautonomous only because the independent variable $s$ appears multiplicatively, and the corresponding boundary value problem:

$$
\theta^{\prime \prime}=-b(L-s) \cos \theta \quad \theta(0)=0, \quad \theta^{\prime}(L)=1
$$


Fig. 1 Equilibrium shapes of a clamped-free Euler inextensible Elastica under uniformly distributed load (pointing upward). On the left, the configuration absolutely minimizing the total energy, on the right, a local minimizer of the energy which is an absolute minimizer in the convex cone

$C:=\left\{\theta \in W^{1,2}: \theta(0)=0\right.$ and

$\theta(s)<0 \quad \forall \quad s \in(0, L]\}$

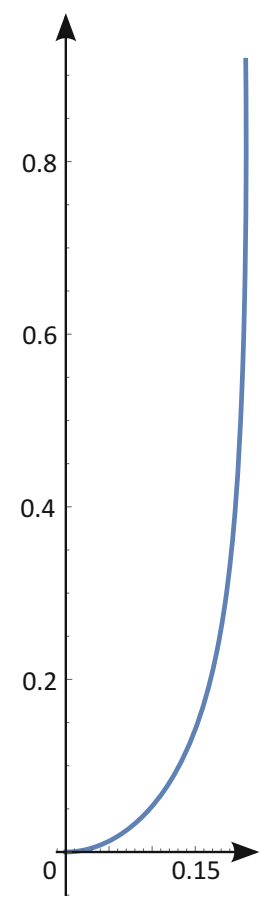

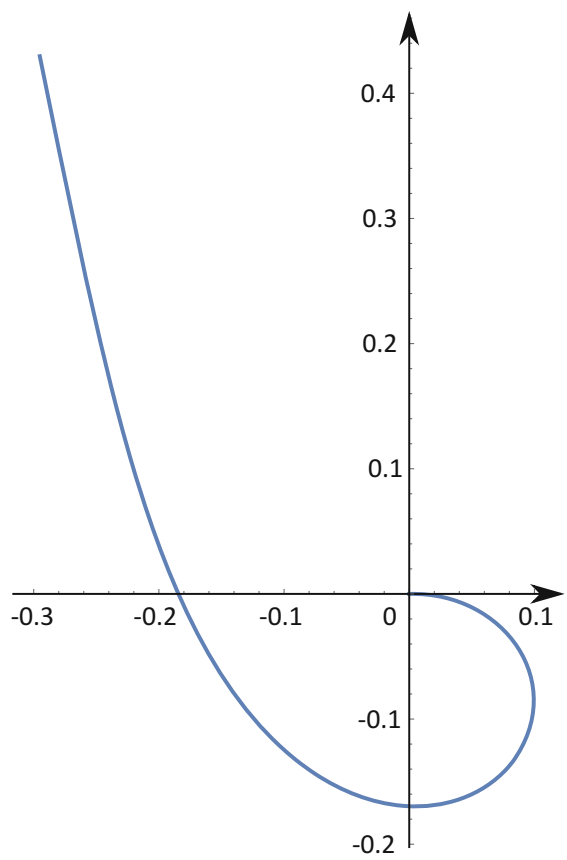

is semilinear and depends on $\theta$ by means of a simple trigonometric function. Since the integrand in (4) is convex with respect to $\theta^{\prime}$ for all $\theta$ and $s$, using a straightforward application of direct method, one can prove the existence of a global minimizer for $E(\theta)$ in $W^{1,2}$; this minimizer corresponds to the deformed shape of the Elastica shown in Fig. 1, left. Moreover, using convexity properties of the set of configurations characterized by having $\theta(s)<0$ for all $s \in(0, L]$, it is possible to prove the existence of "curled" equilibrium configurations, i.e., deformed shapes of the Elastica in which the angle $\theta$ is always negative but at the constrained end (see Fig. 1, right). For the study of the regularity and qualitative properties of the minimizer and of the linear stability of the curled configurations, the reader is referred to Della Corte et al. (2017).

\section{Cross-References}

$>$ Finite Element Methods

\section{References}

Alaoglu L (1940) Weak topologies of normed linear spaces. Ann Math 41:252-267

Axelsson O, Barker VA (2001) Finite element solution of boundary value problems: theory and computation. SIAM, Philadelphia

Ball JM (1976) Convexity conditions and existence theorems in nonlinear elasticity. Arch Ration Mech Anal 63(4):337-403

Ball JM, Mizel VJ (1987) One-dimensional variational problems whose minimizers do not satisfy the EulerLagrange equation. In: Analysis and thermomechanics. Springer, Berlin/Heidelberg, pp 285-348

Banach S (1932) Théorie des opérations linéaires

Belloni M (1995) Interpretation of lavrentiev phenomenon by relaxation: the higher order case. Trans Am Math Soc 347:2011-2023

Berkovitz LD (1974) Lower semicontinuity of integral functionals. Trans Am Math Soc 192:51-57

Bouchitté G, Braides A, Buttazzo G (1995) Relaxation results for some free discontinuity problems. Journal fur die Reine und Angewandte Mathematik 458: $1-18$

Ciarlet PG (1988) Mathematical elasticity. vol. I, volume 20 of studies in mathematics and its applications. North-Holland, Amsterdam

Ciarlet PG (1997) Mathematical elasticity, vol. II: theory of plates, volume 27 of studies in mathematics and its applications. North-Holland, Amsterdam 
Dacorogna B (2007) Direct methods in the calculus of variations, vol 78. Springer, New York

Della Corte A, dell'Isola F, Esposito R, Pulvirenti M (2017) Equilibria of a clamped euler beam (elastica) with distributed load: large deformations. Math Models Methods Appl Sci 27(8):1391-1421

dell'Isola F, Placidi L (2011) Variational principles are a powerful tool also for formulating field theories. In: Variational models and methods in solid and fluid mechanics. Springer, Vienna, pp 1-15

Fonseca I (1987) Variational methods for elastic crystals. Arch Ration Mech Anal 97(3):189-220

Fonseca I, Leoni G (2007) Modern methods in the calculus of variations: $\mathrm{L}^{\wedge} \mathrm{p}$ spaces. Springer, New York

Galerkin BG (1915) Series solution of some problems of elastic equilibrium of rods and plates. Vestn Inzh Tekh 19:897-908

Gelfand IM, Fomin SV (1963) Richard A. Silverman (ed) Calculus of variations. Prentice-Hall, Englewood Cliffs

Kantorovich L, Krylov V (1962) Approximate methods of advanced analysis. Gos Izd Fiz-Math Lit, Leningrad 560

McShane E et al (1940) Necessary conditions in generalized-curve problems of the calculus of variations. Duke Math J 7(1):1-27

Mikhlin SG (1957) Variatsionnye metody v matematicheskoi fizike. Gostekhizdat

Morrey CB (1952) Quasi-convexity and the lower semicontinuity of multiple integrals. Pac J Math 2(1): 25-53

Pedregal P (2000) Variational methods in nonlinear elasticity. SIAM, Philadelphia

Ritz W (1908) Über eine neue Methode zur Lösung gewisser Variations probleme der mathematischen Physik. Journal für die reine und angewandte Mathematik 135(1):1-61

Roubicek T (1997) Relaxation in optimization theory and variational calculus, vol 4. Walter de Gruyter, Berlin

Rudin W (1991) Functional analysis. International series in pure and applied mathematics. McGraw-Hill, New York

Smirnov VI (2014) A course of higher mathematics, vol 62. Elsevier, Burlington

Sobolev SL (1950) Some applications of functional analysis in mathematical physics, vol 90. American Mathematical Society, Providence

Tonelli L (1911) Sui massimi e minimi assoluti del calcolo delle variazioni. Rendiconti del Circolo Matematico di Palermo (1884-1940) 32(1):297-337

Tonelli L (1920) La semicontinuita nel calcolo delle variazioni. Rendiconti del Circolo Matematico di Palermo (1884-1940) 44(1):167-249

Young LC (1937) Generalized curves and the existence of an attained absolute minimum in the calculus of variations. Comptes Rendus de la Société des Sci et des Lettres de Varsovie 30:212-234

Young LC (1938) Necessary conditions in the calculus of variations. Acta Mathematica 69(1):229-258 